\title{
EFICIÊNCIA E EFEITOS SUBLETAIS DE NIM \\ SOBRE Bonagota salubricola (MEYRICK) (LEPIDOPTERA: TORTRICIDAE) ${ }^{1}$
}

\author{
DANIEL BERNARDI ${ }^{2}$, OSCAR ARNALDO BATISTA NETO E SILVA ${ }^{3}$, ODERLEI BERNARDI ${ }^{4}$, \\ ALEXANDRE DA SILVA ${ }^{5}$, UEMERSON SILVA DA CUNHA ${ }^{6}$, MAURO SILVEIRA GARCIA $^{7}$
}

RESUMO - O objetivo deste trabalho foi avaliar a eficiência e os efeitos subletais de um inseticida à base de nim (10 g.L.-1 de azadiractina A) sobre a lagarta-enroladeira Bonagota salubricola em laboratório. Nos bioensaios, foi utilizado o produto à base de azadiractina NeemAzal-T/S ${ }^{\circledR}$ nas concentrações de $0,06 \%$; $0,09 \% ; 0,12 \% ; 0,16 \% ; 0,18 \%$ e $0,20 \%$ do produto comercial (p.c) e uma testemunha (água destilada). A dieta artificial foi cortada em cubos e imersa nas caldas das respectivas concentrações do produto, e, em seguida, lagartas recém-eclodidas foram transferidas para tubos de vidro, contendo os cubos de dieta tratados. Quando a dieta artificial foi tratada com concentração de $0,20 \%$ do produto comercial, houve $100 \%$ da mortalidade aos 6 dias após a inoculação. Por outro lado, as concentrações de 0,16 e $0,18 \%$ prolongaram a fase larval, reduziram a viabilidade de pupas e afetaram negativamente a fecundidade do inseto.

Termos de indexação: Azadiractha indica, nim, lagarta-enroladeira.

\section{EFFICIENCY AND SUBLETHAL EFFECTS OF NEEM ON Bonagota salubricola (MEYRICK) (LEPIDOPTERA: TORTRICIDAE)}

\begin{abstract}
The objective of this study was to evaluate the efficiency of control and sublethal effects of neem insecticide (10 g.L. $\mathrm{L}^{-1}$ of azadirachtin A) to the leafroller Bonagota salubricola at laboratory condition. In the bioassays, it was used an azadirachtin based product (NeemAzal-T/S ${ }^{\circledR}$ ) at concentrations of $0.06 \%$, $0.09 \%, 0.12 \%, 0.16 \%, 0.18 \%$ and $0.20 \%$ of the commercial product (c.p.) and a control (distilled water). The artificial diet was cut in cubes and immersed in the syrups of the respective product concentrations, soon afterwards recently-emerged caterpillars were transferred to glass tubes, containing the diet cubes treated. When the artificial diet was treated with a concentration of $0.20 \%$ of the commercial product, the mortality was $100 \%$ at the $6^{\text {th }}$ day after inoculation. Additionally, concentrations of $0.16 \%$ and $0.18 \%$ extended the larval stages, reduced pupae viability and negatively affected the fecundity of the insect.
\end{abstract}

Index terms: Azadiractha indica, neem, Brazilian apple leafroller.

\footnotetext{
${ }^{1}$ (Trabalho 127-10). Recebido em: 18-05-2010. Aceito para publicação em: 23-03-2011.

${ }^{2}$ Eng. Agr., Mestrando do Depto. de Fitossanidade, FAEM/UFPel, C. P. 354, CEP: 96010-900, Pelotas-RS. E-mail: dbernardi2004@yahoo.com.br

${ }^{3}$ Tecnólogo em Produção de grãos, Doutorando do Depto. de Entomologia e Acarologia, ESALQ/USP, Av. Pádua Dias, 11, CEP: 13418-900, Piracicaba-SP. E-mail: oscar.netto@usp.br

${ }^{4}$ Eng. Agr., Doutorando do Depto. de Entomologia e Acarologia, ESALQ/USP, Av. Pádua Dias, 11, CEP: 13418-900, Piracicaba-SP. E-mail: oderleibernardi@yahoo.com.br

${ }^{5}$ Estudante de Agronomia, Depto. de Fitossanidade, FAEM/UFPel, Caixa Postal 354, CEP: 96010-900, Pelotas-RS. E-mail alexandrepalmitinho@yahoo.com.br

${ }^{6}$ Eng. Agr., Dr., Prof. Depto. de Fitossanidade, FAEM/UFPel, C. P. 354, CEP: 96010-900, Pelotas-RS.E-mail: uscunha@ufpel.edu.br ${ }^{7}$ Eng. Agr., Dr., Prof. Depto. de Fitossanidade, FAEM/UFPel, C. P. 354, CEP: 96010-900, Pelotas-RS. E-mail: garciasmauro@yahoo.com.br
} 


\section{INTRODUÇÃO}

A lagarta-enroladeira Bonagota salubricola (Meyrick, 1937) (Lepidoptera: Tortricidae) é uma praga de grande importância para a cultura da macieira no Brasil (KOVALESKI et al., 1998). Trata-se de um inseto polífago, cujas lagartas danificam a epiderme das frutas de macieira, pereira e pessegueiro, reduzindo seu valor de mercado e, em alguns casos, chegando a inviabilizar sua comercialização. $\mathrm{Na}$ cultura da macieira, os danos de B. salubricola têm sido superiores aos ocasionados pela mosca-dasfrutas Anastrepha fraterculus (Wiedemann) (Diptera: Tephritidae), atingindo cerca de $10 \%$ da produção (KOVALESKI, 1994; BOTTON, 1999).

O controle de B. salubricola é basicamente realizado por produtos químicos, sendo o inseticida clorpirifós-etil o mais eficiente (BOTTON et al., 2000; KOVALESKI; RIBEIRO, 2003). No entanto, recentemente, mediante a utilização de feromônios sexuais sintéticos para o monitoramento da praga nos pomares e a aplicação de inseticidas fosforados para seu controle, as perdas na produção foram reduzidas para 3 a 5\% (KOVALESKI; RIBEIRO, 2003). Apesar da utilização de confusão sexual para o controle do inseto, os produtores ainda têm empregado o controle químico como principal forma de manejo.

Diante da preocupação crescente para racionalizar e/ou substituir o emprego de inseticidas sintéticos, especialmente nos agroecossistemas frutícolas, e principalmente após a implementação da produção integrada de frutas, os inseticidas naturais passaram a ser considerados uma importante alternativa de controle. O uso de plantas inseticidas, seja por meio de extratos, seja até mesmo de substâncias isoladas das mesmas, não é uma técnica recente, pois já era utilizada desde o século passado, principalmente nos países tropicais, antes do advento dos inseticidas sintéticos (VENDRAMIM, 1997; RODRÍGUEZ; VENDRAMIM, 1998). No entanto, em virtude do surgimento, na década de 40, de inseticidas como o DDT, que se mostravam mais eficientes e baratos, houve uma enorme descrença pelos inseticidas naturais. O ressurgimento destes deveu-se à necessidade de se dispor de novos compostos para uso no controle de pragas sem os problemas de contaminação ambiental, resíduos nos alimentos, efeitos prejudiciais sobre organismos benéficos e seleção de populações de insetos resistentes, já que tais características normalmente estão presentes nos inseticidas vegetais (VENDRAMIM, 1997).

Nesse contexto, espécies de meliáceas foram identificadas como as mais promissoras, destacandose Azadiractha indica A. Juss., como alternativa ao controle químico de diversos insetos-praga (MARTINEZ, 2002). Dessa espécie tem sido extraída a azadiractina, que possui propriedades inseticidas, afetando diversas espécies de insetos, cujos extratos têm-se revelado tão potentes quanto os inseticidas comerciais (SCHMUTTERER, 1988; SCHMUTTERER, 1990; MARTINEZ; VAN ENDEM, 2001).

Nesse sentido, objetivou-se avaliar a eficiência e os efeitos subletais de um inseticida à base de azadiractina $\mathrm{A}\left(\mathrm{NeemAzal}-\mathrm{T} / \mathrm{S}^{\circledR}\right)$ sobre a lagartaenroladeira B. salubricola em laboratório.

\section{MATERIAIS E MÉTODOS}

Para a realização dos bioensaios, foi utilizada uma dieta artificial à base de feijão-carioca e levedura de cerveja, que após o preparo foi acondicionada em caixas plásticas $\left(G_{e r b o x}{ }^{\circledR}\right)$, e posteriormente cortada em cubos de 1,0 cm de lado, os quais foram imersos nas caldas dos respectivos tratamentos, por aproximadamente 3 segundos. Em seguinda, os cubos foram colocados sobre folhas de papelfiltro por 2 minutos para a eliminação do excesso de umidade. Posteriormente, em câmara asséptica, lagartas recém-eclodidas foram transferidas, com o auxílio de um pincel fino, para tubos de vidro (2,5 x $8,5 \mathrm{~cm}$ ) previamente esterilizados e tamponados com algodão hidrófobo, contendo os cubos de dieta tratados. Após a inoculação, os tubos de vidro foram acondicionados em câmara climatizada à temperatura de $25 \pm 2{ }^{\circ} \mathrm{C}$, umidade relativa do ar de $70 \pm 10 \%$ e fotofase de 14 horas.

O produto testado foi o NeemAzal- $\mathrm{T} / \mathrm{S}^{\circledR}(10$ g. $\mathrm{L}^{-1}$ de azadiractina A) nas seguintes concentrações: $0,06 \% ; 0,09 \% ; 0,12 ; 0,16 ; 0,18$ e $0,20 \%$ do produto comercial (p.c) e um tratamento-controle (água destilada).

Os seguintes parâmetros biológicos foram avaliados: mortalidade diária até 12 dias após a inoculação (DAI), duração e viabilidade das fases de ovo, larva e pupa, razão sexual, peso de pupas com $24 \mathrm{~h}$ de idade, período de oviposição, fecundidade e longevidade dos adultos.

Nas concentrações em que houve lagartas sobreviventes, a duração e a viabilidade das fases de larva, pré-pupa e pupa foram determinadas por meio de observações diárias. Assim que se formavam as pupas, procedia-se à retirada e ao acondicionamento das mesmas em tubos de vidro de fundo chato $(2,5 \mathrm{x}$ $8,5 \mathrm{~cm}$ ), contendo no interior papel-filtro umedecido com água destilada e foram vedados com filme plástico. A separação dos sexos foi baseada em características morfológicas dos adultos (BENTANCOURT; SCATONI, 1995) e a razão sexual (rs) calculada, 
utilizando-se da fórmula: $r s=\left(n^{\circ}\right.$ de fêmeas $/ n^{\circ}$ de machos $+n^{\circ}$ de fêmeas). A fecundidade, fertilidade e longevidade de adultos de $B$. salubricola foram avaliadas individualizando-se 10 casais por tratamento, em gaiolas (copos plásticos) transparentes com capacidade de $300 \mathrm{~mL}$. As gaiolas foram dispostas sobre placas de Petri (10 cm de diâmetro), sendo os adultos alimentados com solução de mel a 10\%. A cada dois dias, as posturas foram retiradas e, após contagem, incubadas em tubos de vidro $(2,5 \times 8,5$ $\mathrm{cm})$ contendo no interior papel-filtro umedecido com água destilada e foram vedados com filme plástico para a avaliação da viabilidade.

$\mathrm{O}$ delineamento experimental foi o inteiramente casualizado, com 10 repetições, cada uma composta por quatro tubos, contendo três lagartas/ tubo, totalizando 120 indivíduos por tratamento. Os dados de mortalidade foram corrigidos levando-se em consideração a mortalidade no tratamento-controle (ABBOTT, 1925) e submetidos à análise de Probit para obtenção da $\mathrm{CL}_{50}(\mathrm{CL}$ - Concentração Letal) e respectivos intervalos de confiança (IC 95\%), utilizando o programa Polo-PC (LEORA SOFTWARE, 1987). Os dados foram submetidos à análise de variância, utilizando o software SISVAR (FERREIRA, 2003). Nos casos em que o teste F da Anova foi significativo, compararam-se as médias pelo teste de Tukey $(\mathrm{P} \leq 0,05)$.

\section{RESULTADOS E DISCUSSÃO}

A $\mathrm{CL}_{50}$ e $\mathrm{CL}_{90}$ para B. salubricola, quando exposta a dieta artificial tratada com NeemAzal-T/ $\mathrm{S}^{\circledR}$, foi de $0,15 \%$ e $0,24 \%$, respectivamente (Tabela 1). No entanto, na concentração $0,20 \%$, o produto NeemAzal-T/S ${ }^{\circledR}$ ocasionou mortalidade total dos insetos aos 6 dias após a inoculação (DAI), enquanto, nas demais concentrações, a mortalidade variou de $12,5 \%(0,06 \%$ p.c. $)$ a $55,8 \%(0,18 \%$ p.c. $)$. Aos 12 DAI, constatou-se pequena variação em termos de eficiência de controle em relação à avaliação aos 6 DAI, exceto para a concentração de $0,18 \%$, na qual a mortalidade chegou a $75 \%$ (Tabela 2). De modo semelhante, Cunha et al. (2003), avaliando o potencial de NeemAzal-T/S ${ }^{\circledR}$ para controle da traçado-tomateiro Tuta absoluta (Meyrick) (Lepidoptera: Gelechiidae), constataram que o produto formulado a $0,18 \%$ causou mortalidade superior a $90 \%$ das larvas aos 6 dias após a aplicação e mortalidade total quando aplicado a $0,20 \%$.

Além do NeemAzal-T/S ${ }^{\circledR}$, outros produtos à base de azadiractina demonstram ser eficientes para controle de insetos-praga. Bernardi et al. (2010) avaliaram diferentes produtos comerciais à base de nim para controle de Grapholita molesta Busck (Lepidoptera: Torticidade), os quais verificaram mortalidade total aos 3 e 12 DAI, quando as lagartas foram exposta à dieta artificial tratada com NeemAzal-T/ $\mathrm{S}^{\circledR}$ e Dalneem emulsionável ${ }^{\circledR}$, ambos a $0,5 \%$ do p.c. A mortalidade de lagartas nos primeiros instares por produtos à base de nim foi relatada por Rodríguez e Vendramim (1997) para lagartas de Spodoptera frugiperda (J.E. Smith) (Lepidoptera: Noctuidae) criadas em dieta artificial com extrato de sementes de nim $(5 \%)$. A eficiência de produtos à base de nim foi destacada por Góez et al. (2003), os quais constataram que, dentre vários extratos vegetais, aqueles oriundos de $A$. indica estão entre os mais eficientes no controle de diversos lepidópteros-praga. A eficiência de $A$. indica no controle de tortricídeos foi demonstrada por Lowery \& Smirle (2003), os quais verificaram elevada toxicidade de um extrato de sementes de nim a lagartas de Choristoneura rosaceana (Harris) (Lepidoptera: Tortricidae). Adicionalmente, constataram que o extrato, quando aplicado topicamente $(0,5 \mu \mathrm{L} /$ larva de sexto instar), em concentrações de $0,002 \%$ a $0,032 \%$, ocasionou alterações fisiológicas que tornaram os insetos sobreviventes mais suscetíveis aos inseticidas piretroides, carbamatos e organofosforados comumente utilizados para controle da praga em pomares de macieira. Portanto, os autores concluíram que os inseticidas à base de nim poderiam ser ferramentas úteis para o manejo de populações de $C$. rosaceana que apresentaram resistência aos inseticidas sintéticos.

Em relação à duração do período larval, constatou-se um aumento significativo nos tratamentos em que o NeemAzal-T/S $\mathrm{S}^{\circledR}$ foi aplicado a 0,16 ou $0,18 \%$ comparativamente às concentrações de 0,06 e $0,09 \%$ e testemunha (Tabela 2). Rodríguez e Vendramim (1997) observaram que lagartas de $S$. frugiperda tiveram um crescimento mais lento, resultante da ingestão de extratos aquosos das meliáceas A. indica, Melia azedarach e Toona ciliata. Bruce et al. (2004) também verificaram prolongamento da fase de larva de Sesamia calamistis (Hampson) (Lepidoptera: Noctuidae) e Eldana saccharina (Walker) (Lepidoptera: Pyralidae) quando alimentadas com folhas de milho tratadas com óleo de nim, na concentração de 0,009\% de azadiractina. De acordo com Tanzubil e Mccaffery (1990), um dos efeitos dos inseticidas à base de nim é o aumento da duração da fase larval dos insetos devido à presença de inibidores de crescimento, redução na alimentação e/ou menor eficiência de conversão do alimento ingerido. A duração da fase de pupa para todos os tratamentos com NeemAzal-T/S ${ }^{\circledR}$ não diferiu do tratamento-testemunha, ou seja, não foi afetada 
pela presença do produto (Tabela 2). Resultados semelhantes foram obtidos por Torres et al. (2001), que não observaram diferenças significativas na duração do período de pupa de Plutella xylostella $\mathrm{L}$. (Lepidoptera: Plutellidae) quando as lagartas foram inoculadas em discos de couve (Brassica oleracea) imersos em extratos de $A$. indica. Da mesma forma, Morandi-Filho et al. (2006) não constataram diferenças significativas na duração do período de pupa de Argyrotaenia sphaleropa (Meyrick) (Lepidoptera: Tortricidae) quando alimentadas com dieta artificial tratada com Natuneem Agrícola ${ }^{\circledR}$ a 0,25 e $0,50 \%$. Entretanto, De-Ling et al. (2000) observaram um prolongamento no número de dias da fase de pupa de Helicoverpa armigera Hübner (Lepidoptera: Noctuidae) quando as lagartas foram alimentadas com dieta artificial contendo óleo de nim na concentração $0,3 \%$ de azadiractina. Isto demonstra que o efeito de produtos à base de nim tem uma relação com a espécie-alvo e a dose empregada.

A viabilidade de ovos e de larvas foi reduzida significativamente na medida em que aumentou a concentração de NeemAzal-T/S ${ }^{\circledR}$ na dieta artificial (Tabela 3). A $0,18 \%$ do p.c., a viabilidade de ovos e larvas foi de 76,0 e $28,3 \%$, respectivamente. No entanto, a $0,2 \%$ do p.c. $B$. salubricola não completou a fase larval. A viabilidade da fase de pupa foi de aproximadamente $91 \%$ quando o produto foi aplicado à dieta a 0,16 e $0,18 \%$, diferindo significativamente das concentrações de 0,$06 ; 0,09 ; 0,12 \%$ e testemunha, cujas viabilidades foram de 95,$2 ; 98,0$; 96,3 e 98,2\%, respectivamente. Morandi-Filho et al. (2006) também observaram redução da viabilidade da fase de larva (76 e $75 \%$ ) e de pupa (75\%) do tortricídeo A. sphaleropa, quando em contato com Natuneem ${ }^{\circledR}$ a 0,25 e $0,50 \%$, respectivamente.

Em relação ao peso de pupas de machos e fêmeas, não houve diferenças significativas entre os tratamentos (Tabela 3). Resultados estes que corroboram os encontrados por De-Ling et al. (2000), que não verificaram redução no peso de pupas de $H$. armigera quando as lagartas foram alimentadas com dieta artificial contendo extratos aquosos de óleo de nim $(0,3 \%$ de azadiractina). Da mesma forma, Morandi-Filho et al. (2006) também não observaram redução no peso de pupas de $A$. sphaleropa quando alimentadas com dieta artificial contendo extrato aquoso de nim (Natuneen ${ }^{\circledR} 0,25$ e $0,50 \%$ ). Apesar desta observação, outros autores já constataram redução significativa do peso de pupas para outras espécies-praga como $S$. frugiperda alimentadas com dietas contendo extratos vegetais, dentre eles o nim (RODRIGUEZ;VENDRAMIM, 1997; XIE et al., 1994). Segundo os autores, o menor peso foi resultado da inibição da alimentação ou da menor eficiência de conversão de alimento ingerido pelas lagartas devido à presença de substâncias inseticidas na dieta.

A razão sexual em todos os tratamentos foi semelhante, mantendo a proporção próxima a 1:1 entre número de machos e de fêmeas, não havendo alterações em função do aumento da concentração do produto (Tabela 3 ). Resultados semelhantes foram obtidos por Morandi-Filho et al. (2006), que avaliaram aspectos biológicos de $A$. sphaleropa em dieta artificial contendo Natuneem ${ }^{\circledR}$ a 0,25 e $0,50 \%$.

Em relação à longevidade de adultos machos e fêmeas, não se observaram diferenças significativas (Tabela 4), diferentemente dos resultados obtidos por Bruce et al. (2004), que constataram diferenças na longevidade de machos e fêmeas de $S$. calamistis e E. saccharina, quando alimentadas com folhas de milho tratadas com óleo de nim. Morandi-Filho et al. (2006) também observaram diferenças na longevidade de machos e fêmeas de A. sphaleropa, quando alimentadas com dieta artificial tratada com extratos aquosos de Natuneem ${ }^{\circledR}$ a 0,25 e $0,50 \%$.

A duração do período de oviposição de $B$. salubricola reduziu significativamente apenas para os insetos oriundos do tratamento em que a dieta foi tratada com NeemAzal-T/S ${ }^{\circledR}$ a $0,18 \%$. A fecundidade diária e total foi afetada significativamente nas concentrações de 0,16 e $0,18 \%$, sendo reduzida em até 32 e $50 \%$, respectivamente, quando comparada à testemunha (Tabela 4). Estes resultados concordam com os encontrados por Morandi-Filho et al. (2006), que constataram uma redução de $25 \%$ na fecundidade e no período de oviposição de $A$. sphaleropa, quando as lagartas foram alimentadas com dieta artificial contendo Natuneem ${ }^{\circledR}$. Koul (1998) também verificou redução de $50 \%$ da fecundidade e da fertilidade de adultos de Brevicoryne brassicae (Linnaeus) (Hemiptera: Aphididae) que se alimentaram em folhas de couve pulverizadas com extrato de nim, na concentração de 0,3\%. De modo similar, Almeida (2009) demonstrou que as mariposas de A. gemmatalis apresentaram reduzida capacidade de oviposição após a exposição da fase larval às concentrações de $0,005 \%$ e $0,01 \%$ de extratos de sementes de nim. Além de efeitos diretos (mortalidade), os extratos de semente de nim também reduziram a alimentação e o ganho de peso de larvas e pupas, causaram deformidade de pupas, reduziram a fertilidade e provocaram alterações morfológicas nas células do intestino médio e corpo gorduroso das lagartas.

A eficiência do produto à base de nim (Nee$\left.\mathrm{mAzal}-\mathrm{T} / \mathrm{S}^{\circledR}\right)$ no controle de B. salubricola deve-se aos efeitos fagodeterrentes e tóxicos durante diferen- 
tes fases do ciclo biológico da praga. Adicionalmente, segundo Mordue (Luntz) e Nisbet (2000), existe uma larga margem de segurança para o emprego do nim, tanto para o usuário (produtor) quanto para o consumidor. Diante disso, verifica-se que o emprego de inseticidas naturais à base de nim é uma alternativa ao manejo integrado de $B$. salubricola, podendo vir a contribuir para a redução do uso de inseticidas mais agressivos aos organismos benéficos e ao ambiente.

TABELA 1 - Suscetibilidade de Bonagota salubricola exposta à dieta artificial tratada com diferentes concentrações de NeemAzal-T/S ${ }^{\circledR}$.

\begin{tabular}{lcccccc}
\hline Espécie & $n^{\text {a }}$ & Coef. ang. $( \pm \mathrm{EP})$ & $\begin{array}{c}\mathrm{CL}_{50}(\mathrm{IC} 95 \%) \\
\mu \mathrm{L} \cdot \mathrm{mL}^{-1} \text { água }\end{array}$ & $\begin{array}{c}\mathrm{CL}_{90}(\mathrm{IC} 95 \%) \\
\mu L \cdot \mathrm{mL}^{-1} \text { água }\end{array}$ & $\chi^{2}$ & g.1 $^{\mathrm{b}}$ \\
\hline B. salubricola & 720 & $6,30( \pm 0,99)$ & $0,15(0,14-0,16)$ & $0,24(0,22-0,30)$ & 1,17 & 3 \\
\hline
\end{tabular}

${ }^{a}$ número de indivíduos testados. ${ }^{\mathrm{b}}$ graus de liberdade.

TABELA 2 - Mortalidade (\%) de larvas aos 6 e 12 dias após a inoculação (DAI) e duração (dias) dos estágios de larva e pupa de Bonagota salubricola em dieta artificial tratada com NeemAzal-T/S ${ }^{\circledR} \mathrm{em}$ diferentes concentrações.

\begin{tabular}{|c|c|c|c|c|}
\hline \multirow{2}{*}{$\begin{array}{c}\text { Tratamento } \\
\text { (\% p.c) }\end{array}$} & \multicolumn{2}{|c|}{ Mortalidade (\%) } & \multicolumn{2}{|c|}{ Duração do estágio (dias) $)^{1,2}$} \\
\hline & $6 \mathrm{DAI}$ & $12 \mathrm{DAI}$ & Larva & Pupa $^{(\mathrm{ns})}$ \\
\hline Testemunha & $10,0 \pm 1,25 \mathrm{~d}$ & $11,7 \pm 1,70 \mathrm{~d}$ & $\begin{array}{l}20,8 \pm 0,49 b \\
(20-29)[108]\end{array}$ & $\begin{array}{c}10,1 \pm 0,63 \\
(8-14)[105]\end{array}$ \\
\hline 0,06 & $12,5 \pm 2,10 \mathrm{~d}$ & $14,2 \pm 2,18 \mathrm{~d}$ & $\begin{array}{l}20,5 \pm 0,45 b \\
(10-29)[104]\end{array}$ & $\begin{array}{l}10,2 \pm 0,52 \\
(8-14)[98]\end{array}$ \\
\hline 0,09 & $14,2 \pm 3,00 \mathrm{~cd}$ & $17,5 \pm 2,20 \mathrm{~cd}$ & $\begin{array}{c}21,5 \pm 0,47 \mathrm{~b} \\
(19-28)[99]\end{array}$ & $\begin{array}{l}9,9 \pm 0,60 \\
(8-12)[96]\end{array}$ \\
\hline 0,12 & $27,5 \pm 3,30 \mathrm{c}$ & $36,7 \pm 3,00 \mathrm{c}$ & $\begin{array}{c}23,0 \pm 0,56 \mathrm{ab} \\
(20-28)[79]\end{array}$ & $\begin{array}{l}9,9 \pm 0,49 \\
(8-13)[75]\end{array}$ \\
\hline 0,16 & $34,2 \pm 3,18 b c$ & $57,5 \pm 3,08 \mathrm{bc}$ & $\begin{array}{l}25,2 \pm 0,45 a \\
(21-26)[53]\end{array}$ & $\begin{array}{l}9,8 \pm 0,53 \\
(8-15)[48]\end{array}$ \\
\hline 0,18 & $55,8 \pm 3,20 b$ & $75,0 \pm 3,15 b$ & $\begin{array}{c}25,1 \pm 0,49 a \\
(20-28)[33]\end{array}$ & $\begin{array}{l}9,9 \pm 0,24 \\
(8-13)[30]\end{array}$ \\
\hline 0,20 & $100,0 \pm 0,00 \mathrm{a}$ & $100,0 \pm 0,00 \mathrm{a}$ & & \\
\hline
\end{tabular}

${ }^{1}$ Médias $( \pm \mathrm{EP})$ seguidas da mesma letra na coluna não diferem entre si, pelo teste de Tukey $(\mathrm{P} \leq 0,05)$. ns $=$ não significativo.

${ }^{2}$ Valores entre parênteses expressam o intervalo de variação e entre colchetes indicam o número de observações. p.c.: produto comercial

TABELA 3 - Viabilidade dos estágios de ovo, larva, pupa, peso de pupas (machos e fêmeas) e razão sexual de Bonagota salubricola criada em dieta artificial tratada com NeemAzal-T/S ${ }^{\circledR}$ em diferentes concentrações.

\begin{tabular}{|c|c|c|c|c|c|c|}
\hline \multirow{2}{*}{$\begin{array}{c}\text { Tratamento } \\
\text { (\% p.c.) }\end{array}$} & \multicolumn{3}{|c|}{ Viabilidade $(\%)^{1,2}$} & \multicolumn{2}{|c|}{ Peso de pupas $(\mathrm{mg})^{1,2}$} & \multirow{2}{*}{$\frac{\text { Razão }}{\text { sexual }^{(\mathrm{ns})}}$} \\
\hline & Ovo & Larva & Pupa & Macho $^{(\mathrm{ns})}$ & Fêmea $^{(\mathrm{ns})}$ & \\
\hline Testemunha & $\begin{array}{c}92,0 \pm 7,98 \mathrm{a} \\
(262)\end{array}$ & $\begin{array}{c}90,8 \pm 4,98 \mathrm{a} \\
(120)\end{array}$ & $\begin{array}{c}98,2 \pm 4,22 \mathrm{a} \\
(105)\end{array}$ & $15,0 \pm 0,45$ & $21,1 \pm 0,83$ & 0,55 \\
\hline 0,06 & $\begin{array}{c}91,8 \pm 7,11 \mathrm{a} \\
(295)\end{array}$ & $\begin{array}{c}87,4 \pm 4,95 b \\
(120)\end{array}$ & $\begin{array}{c}95,2 \pm 4,17 \mathrm{a} \\
(98)\end{array}$ & $15,0 \pm 0,41$ & $21,0 \pm 0,74$ & 0,45 \\
\hline 0,09 & $\begin{array}{c}90,2 \pm 7,03 \mathrm{a} \\
(276)\end{array}$ & $\begin{array}{c}83,3 \pm 4,01 \mathrm{c} \\
(120)\end{array}$ & $\begin{array}{c}98,0 \pm 4,08 \mathrm{a} \\
(96)\end{array}$ & $16,1 \pm 0,45$ & $20,2 \pm 0,71$ & 0,40 \\
\hline 0,12 & $\begin{array}{c}87,1 \pm 6,54 \mathrm{ab} \\
(267)\end{array}$ & $\begin{array}{c}66,7 \pm 3,87 \mathrm{~d} \\
(120)\end{array}$ & $\begin{array}{c}96,3 \pm 4,11 \mathrm{a} \\
(75)\end{array}$ & $17,2 \pm 0,51$ & $20,8 \pm 0,76$ & 0,55 \\
\hline 0,16 & $\begin{array}{c}85,9 \pm 6,09 \mathrm{ab} \\
(222)\end{array}$ & $\begin{array}{c}44,2 \pm 3,55 \mathrm{e} \\
(120)\end{array}$ & $\begin{array}{c}91,8 \pm 3,95 \mathrm{~b} \\
(48)\end{array}$ & $14,1 \pm 0,40$ & $22,1 \pm 0,89$ & 0,40 \\
\hline 0,18 & $\begin{array}{c}76,0 \pm 6,75 \mathrm{c} \\
(81)\end{array}$ & $\begin{array}{c}28,3 \pm 3,47 \mathrm{f} \\
(120)\end{array}$ & $\begin{array}{c}91,2 \pm 3,78 \mathrm{~b} \\
(30)\end{array}$ & $14,0 \pm 0,40$ & $21,3 \pm 0,76$ & 0,50 \\
\hline
\end{tabular}

${ }^{1}$ Médias ( \pm EP) seguidas da mesma letra na coluna não diferem entre si, pelo teste de Tukey $(\mathrm{P} \leq 0,05)$. ns= não significativo.

${ }^{2}$ Valores entre parênteses indicam o número de observações.p.c.: produto comercial 
TABELA 4 - Longevidade (dias) de machos e fêmeas, período de oviposição (dias) e fecundidade (diária e total) de Bonagota salubricola em dieta artificial tratada com NeemAzal-T/S ${ }^{\circledR}$ em diferentes concentrações.

\begin{tabular}{|c|c|c|c|c|c|}
\hline \multirow{2}{*}{$\begin{array}{l}\text { Tratamento } \\
(\% \text { p.c. })\end{array}$} & \multicolumn{2}{|c|}{ Longevidade (dias) ${ }^{1,2(\mathrm{~ns})}$} & \multirow{2}{*}{$\begin{array}{l}\text { Período de oviposição } \\
\left(_{(\text {dias })^{1,2}}\right.\end{array}$} & \multirow{2}{*}{ Fecundidade diária ${ }^{1,2}$} & \multirow{2}{*}{$\begin{array}{c}\text { Fecundidade } \\
\text { total }^{1,2}\end{array}$} \\
\hline & Machos & Fêmeas & & & \\
\hline Testemunha & $\begin{array}{l}13,2 \pm 1,64 \\
(9-16)[10]\end{array}$ & $\begin{array}{c}13,6 \pm 1,83 \\
(12-15)[10]\end{array}$ & $\begin{array}{l}10,8 \pm 0,67 \mathrm{a} \\
(7-12)[10]\end{array}$ & $\begin{array}{l}25,8 \pm 2,71 \mathrm{a} \\
(6-44)[10]\end{array}$ & $\begin{array}{c}281,6 \pm 21,3 \mathrm{a} \\
(247-336)[10]\end{array}$ \\
\hline 0,06 & $\begin{array}{l}14,3 \pm 2,00 \\
(7-17)[10]\end{array}$ & $\begin{array}{c}13,6 \pm 1,55 \\
(12-16)[10]\end{array}$ & $\begin{array}{l}11,3 \pm 0,69 \mathrm{a} \\
(9-12)[10]\end{array}$ & $\begin{array}{c}24,3 \pm 2,33 \mathrm{a} \\
(5-44)[10]\end{array}$ & $\begin{array}{c}284,0 \pm 23,2 \mathrm{a} \\
(210-336)[10]\end{array}$ \\
\hline 0,09 & $\begin{array}{l}14,3 \pm 1,85 \\
(8-17)[10]\end{array}$ & $\begin{array}{c}13,2 \pm 1,61 \\
(11-16)[10]\end{array}$ & $\begin{array}{l}11,6 \pm 0,71 \mathrm{a} \\
(9-12)[10]\end{array}$ & $\begin{array}{r}23,3 \pm 2,21 \mathrm{a} \\
(4-41)[10]\end{array}$ & $\begin{array}{c}297,2 \pm 28,3 \mathrm{a} \\
(137-337)[10]\end{array}$ \\
\hline 0,12 & $\begin{array}{l}14,3 \pm 1,93 \\
(8-17)[10]\end{array}$ & $\begin{array}{c}12,6 \pm 1,78 \\
(10-16)[10]\end{array}$ & $\begin{array}{l}10,3 \pm 0,65 \mathrm{a} \\
(8-12)[10]\end{array}$ & $\begin{array}{c}24,6 \pm 2,49 \mathrm{a} \\
(7-48)[10]\end{array}$ & $\begin{array}{c}278,6 \pm 21,5 \mathrm{a} \\
(182-327)[10]\end{array}$ \\
\hline 0,16 & $\begin{array}{l}14,0 \pm 1,76 \\
(10-17)[8]\end{array}$ & $\begin{array}{c}13,3 \pm 1,83 \\
(12-14)[10]\end{array}$ & $\begin{array}{c}10,2 \pm 0,65 \mathrm{a} \\
(9-11)[8]\end{array}$ & $19,9 \pm 2,07 \mathrm{~b}(6-38)[8]$ & $\begin{array}{l}227,1 \pm 20,2 \mathrm{~b} \\
(159-243)[8]\end{array}$ \\
\hline 0,18 & $\begin{array}{c}13,8 \pm 1,83 \\
(12-17)[4]\end{array}$ & $\begin{array}{c}12,3 \pm 1,75 \\
(11-13)[10]\end{array}$ & $\begin{array}{c}7,0 \pm 0,51 \mathrm{~b} \\
(6-8)[4]\end{array}$ & $17,5 \pm 1,93$ b (9-33) [4] & $\begin{array}{l}140,0 \pm 19,35 \mathrm{c} \\
(107-184)[4]\end{array}$ \\
\hline
\end{tabular}

${ }^{1}$ Médias $( \pm \mathrm{EP})$ seguidas da mesma letra na coluna não diferem entre si, pelo teste de Tukey $(\mathrm{P}<0,05)$. ns= não significativo. ${ }^{2}$ Valores entre parênteses expressam o intervalo de variação e entre colchetes indicam o número de observações. p.c.: produto comercial.

\section{CONCLUSÕES}

1-O produto à base de nim NeemAzal-T/S ${ }^{\circledR}$ $\left(10\right.$ g.L $L^{-1}$ de azadiractina A) na dieta artificial a $0,2 \%$ do p.c. causa mortalidade total de larvas de Bonagota salubricola. Na concentração de $0,18 \%$ do p.c. há prolongamento da fase larval, redução da viabilidade de pupas e da fecundidade de B. salubricola.

2-O produto NeemAzal-T/S ${ }^{\circledR}\left(10\right.$ g. $\mathrm{L}^{-1}$ de azadiractina $\mathrm{A}$ ) tem grande potencial para o controle de B. salubricola, no entanto, há necessidade de validação dos resultados sob infestações naturais da praga em condições de campo.

\section{REFERÊNCIAS}

ABBOTT, W. S. A method of computing the effectiveness of an insecticide. Journal of Economic Entomology, College Park, v.18, n.2, p.265-266, 1925.

ALMEIDA, G. D. Potencial de produtos derivados de Azadirachta indica no controle de Anticarsia gemmatalis (Lepidoptera: Noctuidae). 2009. $74 \mathrm{f}$. Dissertação (Mestrado em Fitotecnia) - Universidade Federal de Viçosa, Viçosa, 2009.
BENTANCOURT, C. M.; SCATONI, I. B. Lepidópteros de importância económica en el Uruguay (reconocimiento, biología y daños de las plagas agrícolas y forestales). Uruguay: Hemisfério Sur, Facultad de Agronomia, 1995. v.1, 122p.

BERNARDI, O.; BERNARDI, D.; TRECHA, C. O.; JARDIM, E. O.; CUNHA, U. S.; GARCIA, M. S. Eficiência de inseticidas à base de nim no controle de Grapholita molesta (Busck, 1916) (Lepidoptera: Tortricidae). Revista Brasileira de Fruticultura, Jaboticabal, v.32, n.1, p.286-290, 2010.

BOTTON, M. 1999. Bioecologia e controle de Bonagota cranaodes (Meyrick, 1937) (Lepidoptera: Tortricidae) na cultura da macieira. 1999. $73 \mathrm{f}$. Tese (Doutorado) - Escola Superior de Agricultura Luiz de Queiroz, Universidade de São Paulo, Piracicaba, 1999.

BOTTON, M.; NAKANO, O.; KOVALESKI, A. Controle químico da lagarta-enroladeira (Bonagota cranaodes Meyrick, 1937) na cultura da macieira. Pesquisa Agropecuária Brasileira, Brasília, v.35, n.11, p.2139-2144, 2000. 
BRUCE, Y. A.; GOUNOU, S.; CHABI-OLAYE, A.; SMITH, H.; SCHULTHESS, F. The effect of neem (Azadirachta indica A. Juss) oil on oviposition, development and reproductive potentials of Sesamia calamistis Hampson (Lepidoptera: Noctuidae) and Eldana saccharina Walker (Lepidoptera: Pyralidae). Agricultural and Forest Entomology, Midlothian, v.6, n.3, p.223-232, 2004.

CUNHA, U. S.; BOGORNI, P. C.; VENDRAMIM, J. D.; GONÇALVES-GERVÁSIO, R. C. R. Estimativa de concentrações letais de NeemAzal-TS, formulação à base de nim, para a traça-do-tomateiro Tuta absoluta (MEYRICK). In: SIMPÓSIO DE CONTROLE BIOLÓGICO - SICONBIOL, 8., 2003, São Pedro. Anais.... São Pedro: SEB, 2003. p.162.

DE-LING, M. A.; GORDH, G.; ZALUCKI, M. P. Biological effects of azadirachtin on Helicoverpa armigera (Hübner) (Lepidoptera:Noctuidae) fed on cotton and artificial diet. Australian Journal of Entomology, Wembley, v.39, n.4, p.301-304, 2000.

FERREIRA, D. F. SISVAR - Sistema para análise de variância de dados balanceados: Versão 4.3. Lavras: Universidade Federal de Lavras, 2003.

GÓEZ, G. B.; NERI, D. K. P.; CHAVES, J. W. N.; MARACAJÁ, P. B. Efeitos de extratos vegetais no controle de Spodoptera frugiperda (J.E. Smith) (Lepidoptera: Noctuidae). Caatinga, Mossoró, v.16, n.1/2, p.47-49, 2003.

KOUL, O. Effect of neem extracts and azadirachtin on fertility and fecundity of cabbage aphid, Brevicoryne brassicae (L.). Pesticide Research Journal, Perdikis, v.10, n.2, p.258-261, 1998.

KOVALESKI, A. Eficiência dos inseticidas no controle da lagarta-enroladeira (Phtheocroa cranaodes) em condições de laboratório. HortiSul, Pelotas, v.3, n.2, p.30-32, 1994.

KOVALESKI, A.; BOTTON, M.; EIRAS, A. E.; VILELA, E. Lagarta-enroladeira da macieira: bioecologia e controle. Bento Gonçalves: EmbrapaCNPUV, 1998. 22p. (Circular Técnica, 24)

KOVALESKI, A; RIBEIRO, L. G. Manejo de pragas na produção integrada de maçã. In: PROTAS, J. F. S.; SANHUEZA, R. M. V. Produção integrada de frutas: o caso da maçã no Brasil. Bento Gonçalves: Embrapa-CNPUV, 2003. p.61-68.
LEORA SOFTWARE. POLO-PC: a user's guide to probit or logit analysis. Berkeley, 1987. 20p.

LOWERY, D. T.; SMIRLE, M. J. Toxicity of insecticides to obliquebanded leafroller, Choristoneura rosaceana (Lepidoptera: Tortricidae), larvae and adults exposed previously to neem seed oil. Entomologia Experimentalis et Applicata, Edinburgh, v.95, n.2, p.201-207, 2003.

MARTINEZ, S. M.; VAN ENDEM, H. F. Growth disruption, abnormalities and mortality of Spodoptera littoralis caused by azadirachtin. Neotropical Entomology, Londrina, v.30, p.113-125, 2001.

MARTINEZ, S. S. O NIM: natureza, usos múltiplos, produção. Londrina: IAPAR, 2002. 142p.

MORANDI FILHO, W. J.; BOTTON, M.; GRÜTZMACHER, A. D.; NONDILLO, A. Biologia comparada de Agyrotaenia sphaleropa (Meyrick, 1909) (Lepidoptera: Tortricidae) em dieta artificial contendo extratos vegetais. Arquivos do Instituto Biológico, São Paulo, v.73, n.3, p.325-331, 2006.

MORDUE (LUNTZ) A. J.; NISBET A. J. Azadirachtin from the neem tree Azadirachta indica: its action against insects. Anais Sociedade Entomológica do Brasil, Londrina, v.29, p.615-632, 2000.

RODRÍGUEZ H. C.; VENDRAMIM, J. D. Avaliação da bioatividade de extratos aquosos de Meliaceae sobre Spodoptera frugiperda. Revista de Agricultura, Piracicaba, v.72, n.3, p.305-318, 1997.

RODRÍGUEZ, H. C.; VENDRAMIM, J. D. Uso de índices nutricionales para medir el efecto insectistático de extractos de meliáceas sobre Spodoptera frugiperda. Manejo Integrado de Plagas, Turrialba, v.48, p.11-18, 1998.

SCHMUTTERER, H. Potential of azadirachtincontaining pesticides for integrated pest control in developing and industrialized countries. Journal of Insect Physiology, Amsterdam, v.34, p.713-719, 1988.

SCHMUTTERER, H. Properties and potential of natural pesticides from the neem tree, Azadirachta indica. Annual Review of Entomology, Palo Alto, v.35, p.217-297, 1990. 
TANZUBIL, P. B.; MCCAFERRY, A. R. Effects of Azadirachtin and aqueous neem seed extracts on survival, growth and development of the African Armyworm, Spodoptera exempta. Crop Protection, Guildford, v.9, n.5, p.383-386, 1990.

TORRES, A. L.; BARROS, R.; OLIVEIRA, J. V. Efeito de extratos Aquosos de Plantas no Desenvolvimento de Plutella xylostella (L.) (Lepidoptera: Plutellidae). Neotropical Entomology, Londrina, v.30, n.1, p.151-156, 2001.
VENDRAMIM, J. D. Uso de plantas inseticidas no controle de pragas. In: CICLO DE PALESTRAS SOBRE AGRICULTURA ORGÂNICA, 2., 1997, Campinas. Anais... Campinas: Fundação Cargill, 1997. p.64-69.

XIE, Y. S.; ISMAN, M. B.; GUNNING, P.; MACKINNON, S.; ARNASON, J. T.; TAYLOR, D. R.; SANCHEZ, P.; HASBUN, C.; TOWERS, G. H. N. Biological activity of extracts of Trichilia species and the limonoid hirtin against lepidopteran larvae. Biochemical Systematics and Ecology, Oxford, v.22, n.2, p.129-136, 1994. 